LBL-37439 Rev.

UC-401

\title{
An Orthotropic Source of Thermal Atoms
}

Timothy Dinneen, Albert Ghiorso, and Harvey Gould

Chemical Sciences Division

Lawrence Berkeley Laboratory

University of California

Berkeley, California 94720

July 1995

This work was supported by the Director, Office of Energy Research, Office of Basic Energy Sciences, Chemical Sciences Division, of the U.S. Department of Energy under Contract No. DE-AC03-76SF00098. 


\section{DISCLAIMER}

This report was prepared as an account of work sponsored by an agency of the United States Government. Neither the United States Government nor any agency thereof, nor any of their employees, make any warranty, express or implied, or assumes any legal liability or responsibility for the accuracy, completeness, or usefulness of any information, apparatus, product, or process disclosed, or represents that its use would not infringe privately owned rights. Reference herein to any specific commercial product, process, or service by trade name, trademark, manufacturer, or otherwise does not necessarily constitute or imply its endorsement, recommendation, or favoring by the United States Government or any agency thereof. The views and opinions of authors expressed herein do not necessarily state or reflect those of the United States Government or any agency thereof. 


\section{DISCLAIMER}

Portions of this document may be illegible in electronic image products. Images are produced from the best available original document. 


\title{
AN ORTHOTROPIC SOURCE OF THERMAL ATOMS
}

Timothy Dinneen, Albert Ghiorso and Harvey Gould

MS 71-259, Lawrence Berkeley National Laboratory,

University of California, Berkeley, CA 94720

\begin{abstract}
A source of thermal atoms that emits $100 \%$ of its atoms into a narrow beam with small angular divergence is described. It uses both surface ionization and surface neutralization in conjunction with electric fields to selectively emit a highly directional (orthotropic) beam of neutral atoms. The ion recycling process can be modulated electronically and lends itself to scanning. This orthotropic source is ideal for the efficient use of rare atomic species, well suited for atomic clocks, essential in the efficient delivery of radioactive atoms to optical traps, and has potential to produce ultra high intensity beams of stable atoms.
\end{abstract}


For the past 80 years atomic beams have been one of the principal research tools in physics and chemistry $(1,2)$. Atomic beams have been used to determine atomic structure (3), to discover the first quantum electrodynamic effects (4), to measure the spins and magnetic moments of nuclei (5), to study chemical reactions (6) and surfaces (7), and for as diverse applications as epitaxy (8) and atomic clocks (9). Loading short lived radioactive atoms into laser traps is another, very recent, application that is being developed to search for physics beyond the standard model with experiments such as: atomic parity non conservation (10); beta decay asymmetry (11); and the search for a permanent (electron) electric dipole moment $(12,13)$.

Laser traps are very shallow and loading is accomplished by either laser slowing a beam of atoms to the capture velocity of the trap (14), or injecting atoms into a cell with a non-stick coating where capture occurs continuously from the slower atoms in the tail of the Boltzmann distribution (15). In either case, for radioactive atoms, a highly directional beam needs to be produced from a very limited number of atoms - a requirement not well met by conventional collimated atomic beam sources because almost all of the atoms are lost at the collimators. In laser slowing, an atom moving at a thermal velocity of several hundred meters per second can be brought to a stop in a distance of about one meter. To remain within the diameter of the laser beam over this distance requires a high degree of directionality in the atomic beam. For Boltzmann tail capture, only one of several thousand wall bounces results in a slow atom. Consequently, only a small opening in the cell can be tolerated for atom insertion and a highly directional beam is needed to efficiently fill the cell.

In this report we describe a source that produces a highly directional (orthotropic) beam of neutral alkali atoms without the use of external apertures. Our prototype source generates a beam whose forward flux efficiency is 100 times greater than a standard effusive source and fully confined to within an angle of 9 degrees of the axis. The angular confinement is defined by the source geometry and a source having an angular width of less than 1 degree (an increase in the forward flux by a factor of 10,000) is entirely feasible. The orthotropic source can be electrically modulated and has application to atom imaging, atomic clocks and atomic beam experiments.

A schematic of the orthotropic oven is shown in Fig. 1. The interior surface is a high work function metal which, when hot, evaporates alkali atoms as positive ions. The ions are attracted to a negatively biased neutralizer located opposite the exit aperture. The neutralizer is a low work function metal (also hot) from which the ions re-evaporate as neutral atoms. The neutral atoms must either leave through the exit aperture or strike the walls. If the atoms strike the walls they are again ionized and return to the biased neutralizer. If they leave through the exit aperture, they form a beam whose size and divergence is defined solely by the geometry of the neutralizer and aperture.

To test this principle we built a prototype as shown in Fig 1 . It has a $0.5 \mathrm{~cm}$ diameter exit aperture, a $0.3 \mathrm{~cm}$ diameter neutralizer, an interior diameter of $2.0 \mathrm{~cm}$ and a neutralizer to exit aperture distance of $2.3 \mathrm{~cm}$, producing a beam with all the atoms confined within an angle of $\pm 150 \mathrm{mR}$ ( \pm 9 degrees) to the axis. The body is a stainless steel cube (overall size $4 \mathrm{~cm}$ ) with platinum (work function $5.65 \mathrm{eV}$ (16) ) sputtered on the inside surfaces. A small aspect ratio is used to ensure penetration of the electric field 
into the volume and a tapered end cap, that fits flush on the bottom, allows access to the interior. The neutralizer is made of yttrium (work function $3.4 \mathrm{eV}$ ) and rests slightly above the rear surface of the source on an aluminum oxide spacer. The oven is heated by passing current through tantalum wire threaded through insulators in the body and can reach a maximum of $950^{\circ} \mathrm{C}$.

The orthotropic oven was tested using francium. At atomic number 87, francium is the heaviest alkali in the periodic table. It has no stable or long-lived isotopes. We obtained ${ }^{221} \mathrm{Fr}\left(\mathrm{t}_{1 / 2}=5 \mathrm{~m}\right)$, continuously from the alpha decay of $225 \mathrm{Ac}$. The actinium was prepared by electrostatic collection, in a helium atmosphere, of $225 \mathrm{Ra}$ recoils (17) emitted in the alpha decay of ${ }^{229} \mathrm{Th}\left(\mathrm{t}_{1 / 2}=7300 \mathrm{Y}\right)$ from a thin source $(18)$. The ${ }^{225} \mathrm{Ra}$ which beta decays $\left(t_{1 / 2}=15 d\right)$ to actinium, was collected on a nickel foil disk and mounted in the rear of the orthotropic source. It produced approximately $10^{4} \mathrm{Fr}$ atoms per second with a combined $\mathrm{Ra} / \mathrm{Ac}$ half life of 25 days.

To measure the angular distribution, the orthotropic oven was heated to $750^{\circ} \mathrm{C}$ and the emitted francium atoms allowed to accumulate on a catcher for 45 minutes. The collected francium decays ${ }^{221} \mathrm{Fr} \rightarrow>217 \mathrm{At} \rightarrow 213 \mathrm{Bi}$, the latter having a 45 minute half-life which allows a large number of bismuth atoms to accumulate. The $213 \mathrm{Bi}$ atoms were then detected by the $8.38 \mathrm{MeV}$ alpha emitted in the decay of $213 \mathrm{Po}\left(\mathrm{t}_{1 / 2}=160 \mu \mathrm{s}\right)$ following the beta decay of $213 \mathrm{Bi}$. The catcher was stepped over a collimated surface barrier detector to map the distribution. Fig. 2 shows the distribution and demonstrates that a low divergence francium beam is emerging from the orthotropic source.

The total flux of neutral atoms was calculated by measuring the flux of francium atoms in the central peak as a function of temperature, and combining the result with the angular distribution measurements. For each temperature we verified the angular distribution by measuring the activity on the catcher at both the center and a position corresponding to an angular divergence of $>150 \mathrm{mR}$. (The ${ }^{221} \mathrm{Fr}$ half-life is too short to permit a complete scan for each data point.) In all the measurements no francium (5.6 MeV alpha) was detected beyond $150 \mathrm{mR}$. The total flux is shown as a function of temperature in figure 3. At $950^{\circ} \mathrm{C}$, the maximum temperature of the prototype source, the flux is still increasing rapidly and the yield is $15 \%$. The experiment was repeated with both positive and negative biases on the catcher giving identical results, indicating that the beam is entirely neutral.

To reach a $100 \%$ yield we need to understand the cycling time within the oven and also the effects of diffusion in the oven walls. If these times are too long the atoms will decay inside the oven. The cycling time within the oven depends on the sticking time of atoms with the walls and the number of attempts (100 on average) made before escape. To measure the sticking time we first collected the recoiling ${ }^{221} \mathrm{Fr}_{\mathrm{r}}$ directly onto the surface of $\mathrm{Ni}, \mathrm{Pt}$ and $\mathrm{Y}$ foils using the electrostatic collection technique described earlier. The foils were then heated and the evaporation time measured. These times were found to be very short $\left(<1\right.$ second at $\left.700^{\circ} \mathrm{C}\right)$ and do not contribute to the loss in the prototype source.

Diffusion arises in our orthotropic oven because we begin with $225 \mathrm{Ra} / 225 \mathrm{Ac}$ on the nickel source and not francium. In the alpha decay of ${ }^{225} \mathrm{Ac}$ the francium daughter gains enough kinetic energy to bury itself to a depth of about $400 \AA$ in the platinum coating or the nickel foil. To measure the diffusion time we performed tests with foils of $\mathrm{Pt}$ and $\mathrm{Ni}$ on which ${ }^{225} \mathrm{Ra} / 225 \mathrm{Ac}$ sources were grown. The foils were heated and the release time and 
yield of the implanted francium were measured as a function of temperature. At $950^{\circ} \mathrm{C}$ we find that approximately $25 \%$ of the francium is released from a $\mathrm{Pt}$ foil and about $35 \%$ from a $\mathrm{Ni}$ foil while at higher temperatures a much greater fraction is released. Although slightly greater than the oven yield, the dependence of the released fraction with temperature is very similar to the that found in the orthotropic source, hence a design with increased source temperature should greatly increase the flux.

A comparison between the orthotropic source, a channeled source of similar dimensions, and an effusive source is made in Fig. 4. It assumes that all sources emit the same number of atoms. This is the case here, for example, when all run at the same temperature and are limited by the initial diffusion of the francium atoms. Utilization of the atoms by this $150 \mathrm{mR}$ orthotropic source is a factor of 10 more efficient than for a channeled source and a factor of 100 more efficient than for an effusive source. Since the efficiency scales inversely as the square of the divergence angle, we expect to realize tremendous gains in a source with a far smaller divergence.

The orthotropic source is uniquely suited for efficiently loading short lived radioactive atoms into an optical trap. A thermal beam from an orthotropic source with a $10 \mathrm{mR}$ divergence could be laser slowed in a Zeeman slowing section, and trapped with $20 \%$ efficiency. With an effusive source only about $10^{-5}$ to $10^{-4}$ of the atoms can be trapped $(10,11)$. The efficiency of an orthotropic oven will allow traps to be filled with sufficient numbers $\left(>10^{7}\right)$ to allow experiments, using radioactive alkali atoms, to study parity nonconservation, beta decay asymmetry and to search for a permanent electron electric dipole moment.

By adding a second stage, the orthotropic source could be used to make high flux beams of stable atoms. The second stage could be a separately heated oven that controls the rate of flow into the orthotropic source. The orthotropic stage then acts as a beam brightener by compressing all the flux into a smaller angle without loss. The second stage is needed as a reservoir to store the large number of atoms for such a beam and also to prevent the atoms from coating the high work function walls and quenching the ionization in the orthotropic section. With no more than $10^{14}$ atoms $/ \mathrm{cm}^{2}$ on the oven walls and a temperature that gave an atom sitting time of $1 \mathrm{~ms}$, this prototype source would allow a flux of $10^{15} / \mathrm{s}$ into $150 \mathrm{mR}$; which compares favorably with a standard effusive source of $5 \times 10^{16}$ molecule $\mathrm{sr}^{-1} \mathrm{~s}^{-1}$. If optimized, this might be a way to produce an ultra high brightness source without excessive loading of the vacuum system.

A very useful feature for the orthotropic source is to operate in a pulsed mode. We were able to turn off the source by simply reversing the polarity of the bias on the neutralizer. Two very useful applications result: In situations where a radioactive isotope is produced continuously, a large number of atoms can be accumulated in the oven and then quickly pulsed out. This is very appealing for laser trapping and cooling of rare atoms where pulsed loading times could be much shorter than the trap loss times; Another application is for chopped beams. With the orthotropic source chopping can be done electronically, with the pulse duration and repetition period varied from pulse to pulse. An orthotropic source with multiple neutralizers could be used to make a scanning atomic beam.

We have so far considered only orthotropic sources with very high ionization efficiencies. However, the directed flux of the source is related to the ionization efficiency 
so that even a low ionization efficiency can significantly enhance a low-divergence beam when there are a limited number of atoms available. For example, an experiment that requires a $10 \mathrm{mR}$ beam will typically receive only $10^{-4}$ of the total flux of an effusive source. If we can replace the oven with an orthotropic source designed for $10 \mathrm{mR}$ then even a $1 \%$ ionization fraction from the interior walls will result in $1 \%$ of the output going into $10 \mathrm{mR}$. This is an increase of two orders of magnitude in the required direction.

The $1 \%$ ionization example here corresponds to a situation where, at $950{ }^{\circ} \mathrm{C}$, the ionization potential of the atom is $0.4 \mathrm{eV}$ greater than the work function of the wall surface. The ratio, $\mathrm{n}_{+} / \mathrm{n}_{\mathrm{o}}$ of ions to atoms evaporated from a surface of work function $\Phi$ at a temperature, $\mathrm{T}(\mathrm{O} \mathrm{K})$ where the atoms have an ionization potential, $\mathrm{I}$ is given by the Langmuir Saha eq. (19):

$$
\mathrm{n}_{+} / \mathrm{n}_{\mathrm{o}}=\omega_{+} / \omega_{0} \exp [\varepsilon(\Phi-\mathrm{I}) / \mathrm{kT}]
$$

where $\omega_{+} / \omega_{0}$ is the ratio of statistical weights, which for alkali atoms is equal to $1 / 2$ and $\varepsilon$ is the charge on the electron. Fig. 5 shows $n_{+} / n_{0}$ as a function of $\Phi-I$ at $700^{\circ} \mathrm{C}$, $950^{\circ} \mathrm{C}$ and $1200^{\circ} \mathrm{C}$. The $1 \%$ ionization fraction extends the range of atoms for which an orthotropic source with platinum coated walls is useful, from the alkalis, $\mathrm{Ba}, \mathrm{Ra}, \mathrm{Ac}$ and a few lanthanides to those with ionization potentials up to $6.1 \mathrm{eV}$ or higher. This includes Al, $\mathrm{Ca}, \mathrm{Ga}, \mathrm{Sr}, \mathrm{In}$, most of the lanthanides, $\mathrm{Tl}, \mathrm{Th}, \mathrm{Pa}, \mathrm{Pu}, \mathrm{Am}$, and $\mathrm{Cm}$. Beams of atoms with even higher ionization potential may be possible. A tungsten oxide surface has been used to efficiently ionize thallium (20) (ionization potential $6.1 \mathrm{eV}$ ) for extended periods at $900{ }^{\circ} \mathrm{C}-1000^{\circ} \mathrm{C}$.

To summarize, we have built a highly directional and highly efficient neutral atom source. This orthotropic source can be exploited for experiments in nuclear, atomic and chemical physics. Its efficient use of material makes it ideal for use with radioactive atoms, separated isotopes, and applications where it is important to limit the amount of beam scattered or deposited in the apparatus. Specific examples include: efficient loading of short lived radioactive atoms into optical traps for experiments such as parity non conservation, beta decay asymmetry and the search for a permanent (electron) electric dipole moment; High brightness, long lived sources of stable atoms for atomic clocks; ultra high brightness sources with low vacuum loading.

21

1. L. Dunoyer, Le Radium 8, 142, 1911.

2. See, for example, G. Scoles, Ed., Atomic and Molecular Beam Methods (Oxford University Press, New York, 1988).

3. See, for example, N.F. Ramsey, Molecular Beams (Oxford University Press, London, 1956).

4 W.E. Lamb Jr. and R.C. Retherford, Phys. Rev. 72, 241 (1947); S. Koening, A.S. Prodell, and P. Kush, Phys. Rev. 83, 687 (1951); 88, 191 (1952).

5. I.I. Rabi, J.R. Zacharias, S. Millman, Phys. Rev. 53, 318 (1938); H. Kopfermann, Nuclear Moments (Academic Press, New York, 1958).

6. E.H. Taylor and S. Datz J. Chem. Phys. 231711 (1955); See also Ref. 2. 
7 W.G. Kaenders et al., Nature, 375, 214 (1995).

8 See, for example, E. Kasper, J.C. Bean, Silicon-Molecular Beam Epitaxy (CRC Press, Boca Raton 1988); See also Ref. 2.

9. W.M. Itano and N. Ramsey, Sci. Am. 269, 56 (July 1993).

10. G. Gwinner et al., Phys. Rev. Lett. 72, 3795 (1994).

11. Z-T. Lu et al., Phys. Rev. Lett. 72, 3791 (1994).

12. M. Bijlsma, B.J. Verhaar, D.J. Heinzen, Phys. Rev. A49, 4285 (1994)

13. T.P. Dinneen, H.A. Gould, A. Ghiorso, Bull. Am. Phys. Soc. 40, 1278 (1995).

14. E.L. Raab et al., Phys. Rev. Lett. 59, 2631 (1987).

15. C. Monroe et al., Phys. Rev. Lett. 65, 1571 (1990).

16. D.E. Eastman, Phys. Rev. B2, 1 (1970).

17. A. Ghiorso, private communication.

18. The source was prepared by Isotope Products Laboratory, Burbank, CA from thorium supplied by Brookhaven National Laboratory.

19. I. Langmuir, K.H. Kingdon, Proc.. Roy. Soc. (London) A107, 61 (1925); M.J. Copley, T.E. Phipps, Phys. Rev. 48, 960 (1935).

20. R.E. Beehler, D.J. Glaze, IEEE Transactions in Instrument Science IM-15, 55 (1966).

21. We thank Otto Bischof and Jason Maddi for assistance in testing the orthotropic source, Tony Freeman, and Alan Lyon for assistance in its design and Bob Giaque for timely materials analysis. This work was supported by the Director, Office of Energy Research, Office of Basic Energy Sciences, Division of Chemical Sciences, of the U.S. Department of Energy under contract No. DE-AC-03-76SF00098. 
Fig. 1. Schematic of the Orthotropic source. Neutral atoms are shown emitting in all directions from the neutralizer, some exit from the oven while the remainder hit the walls. Each atom that hits the wall becomes ionized and is returned electrostatically to the neutralizer. An atom may cycle from neutralizer to ionizer and back many times before finally leaving the oven. The result is a very directional and highly efficient source.

Fig. 2. Measured angular distribution of francium atoms from the prototype orthotropic source. The data represent flux through a narrow slit as it is scanned across the source. The solid line shows the expected angular distribution based on the geometry of the source. All of the data points can be seen to lie within $150 \mathrm{mR}$ ( 9 degrees) of the center.

Fig. 3. Temperature dependence of the total flux from our orthotropic source. The oven could not be heated above $950^{\circ} \mathrm{C}$ but the flux is expected to increase further with temperature. The error bars represent statistical fluctuations in the measurements. Systematic errors contribute another $20 \%$ to the measurement, arising mainly from uncertainty in the Fr source activity.

Fig. 4. Normalized integrated angular flux versus angle calculated for our prototype orthotropic source (A), a channeled source (B) and an effusive source (C). An orthotropic source can be designed to put all of its flux into a much smaller solid angle than does our prototype.

Fig. 5. Ionization fraction of alkali atoms evaporating from a surface is plotted as a function of the difference between the ionization potential of the atom and the work function of the surface. Sufficient ionization can occur for the orthotropic source to be of use even when the work function is greater than the ionization potential. 


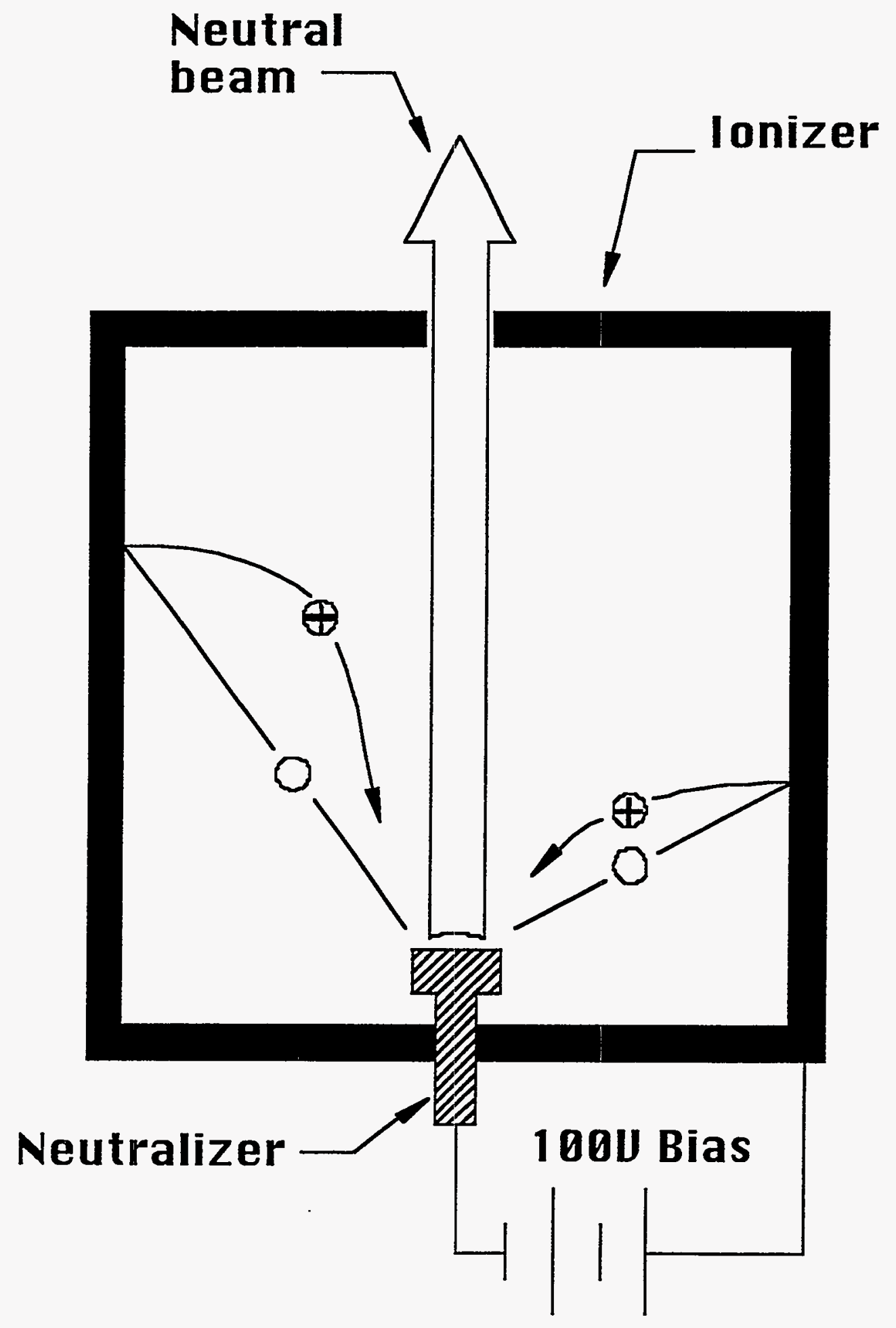




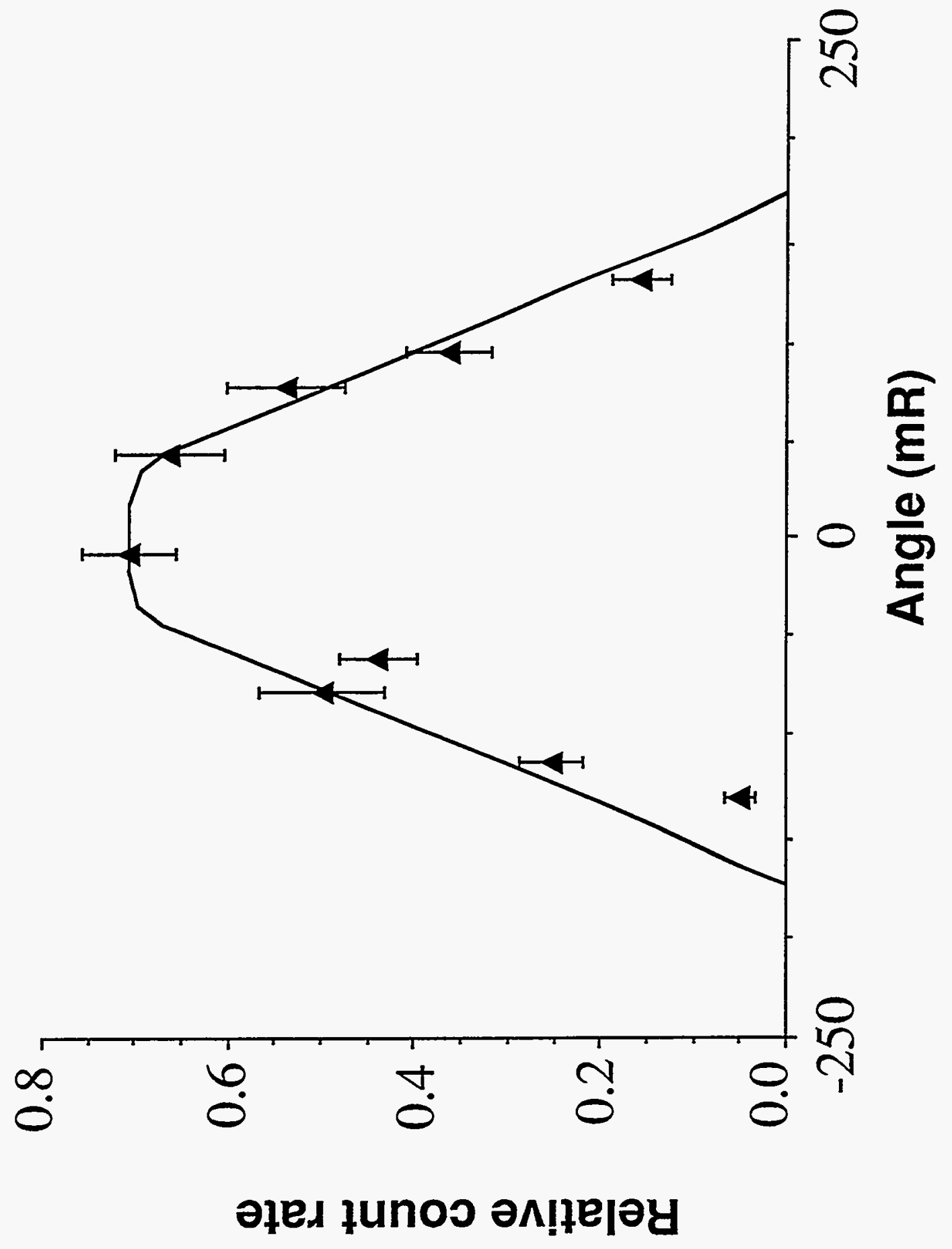




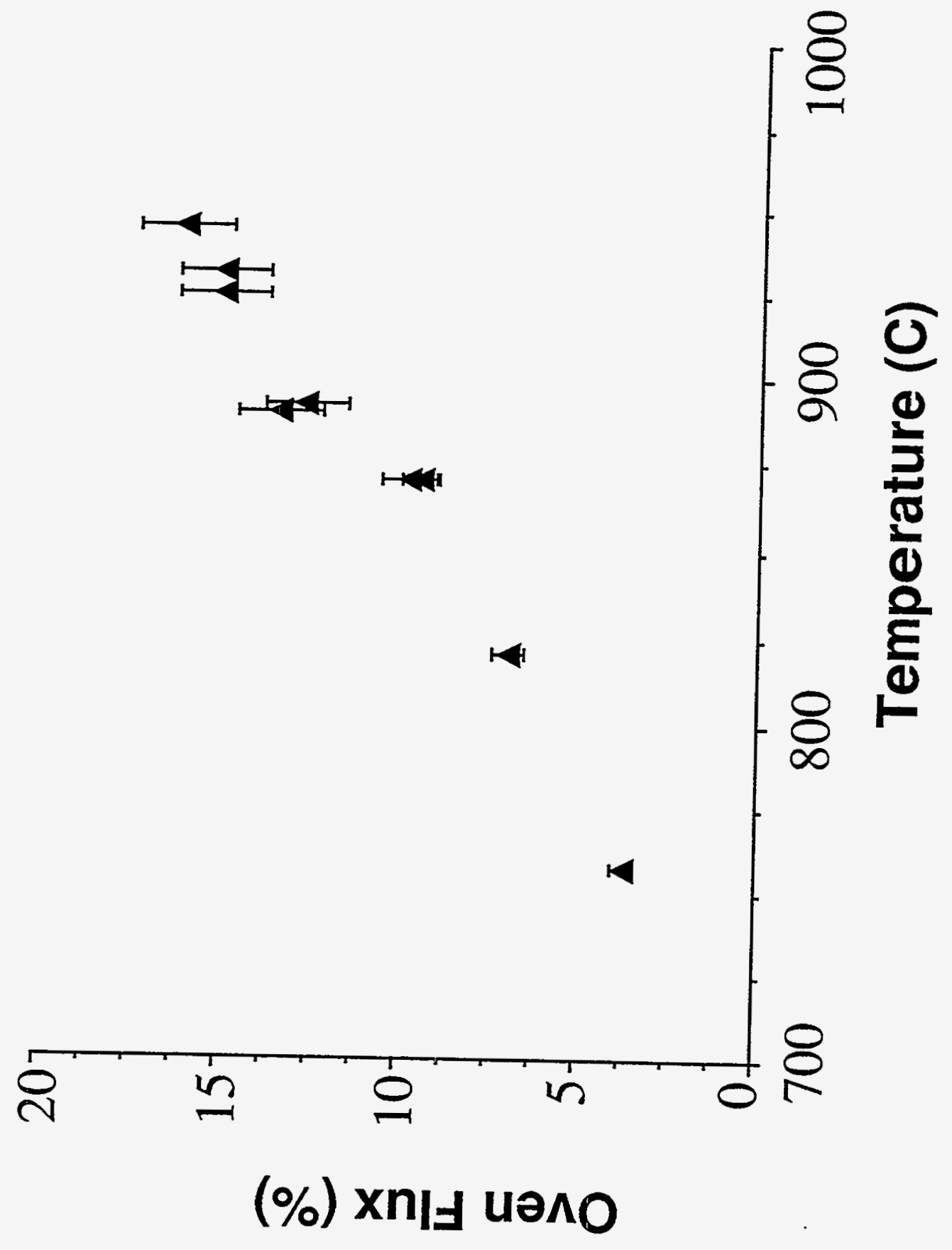




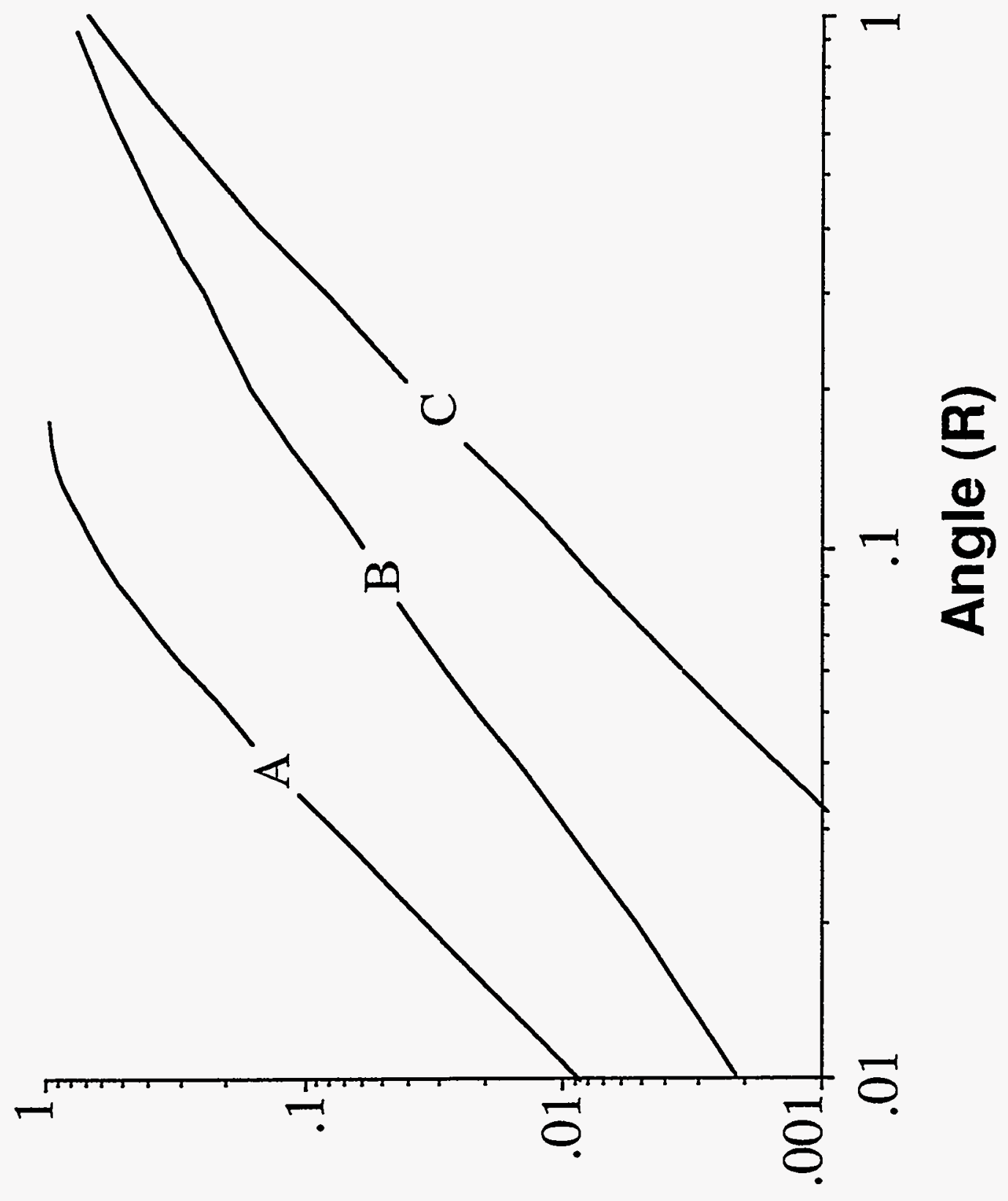

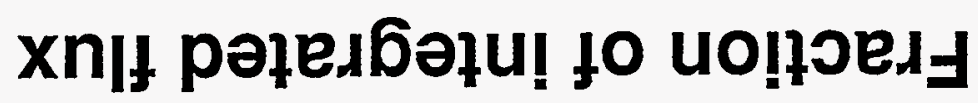




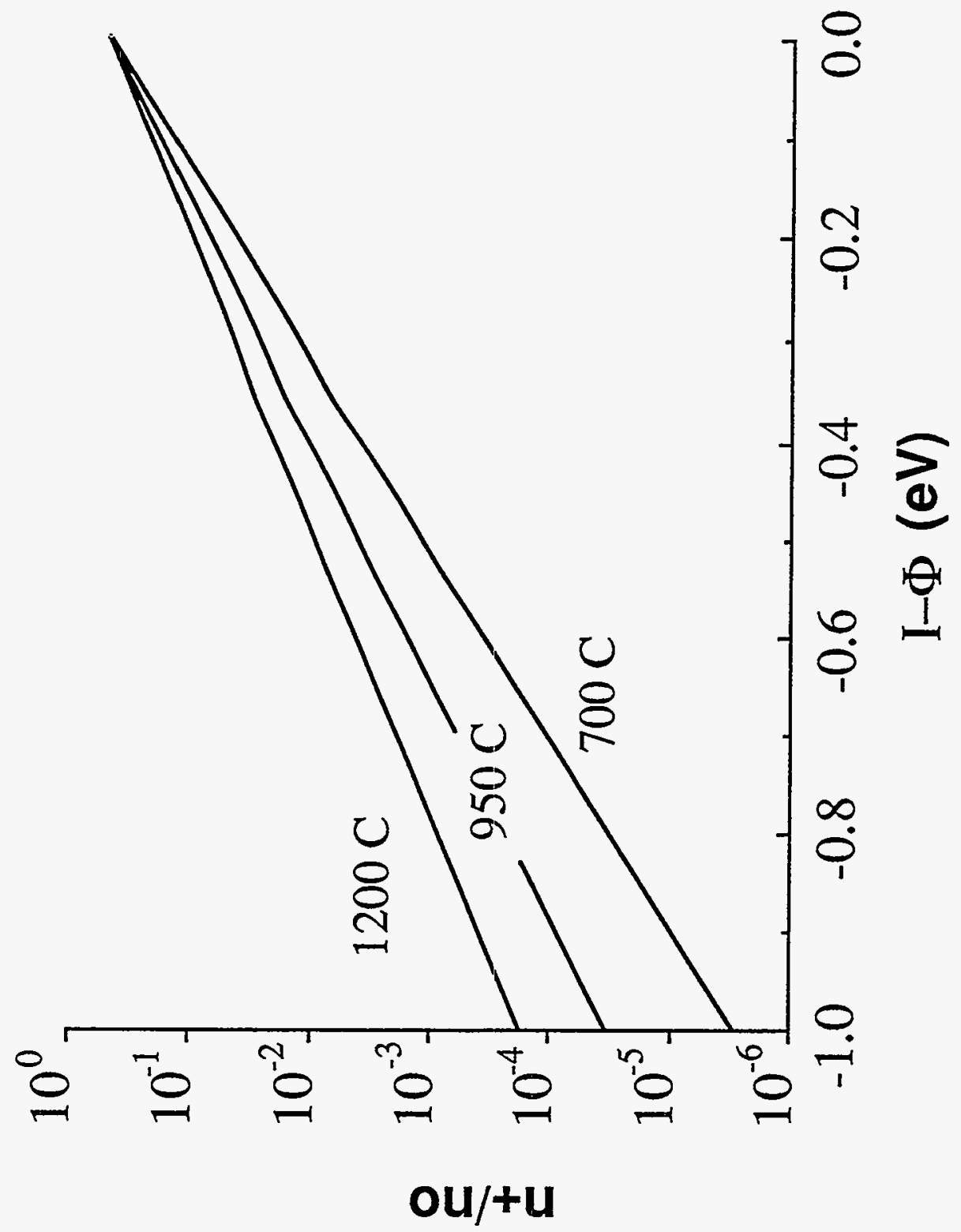

